\title{
New perspective on the $U(n)$ Wigner-Racah calculus: I. Vector coherent state theory and construction of Gel'fand bases
}

\author{
K T Hecht $\dagger$, R Le Blanc $\dagger$ and D J Rowe $\dagger$ \\ † Department of Physics, University of Michigan, Ann Arbor, Michigan 48109, USA \\ $\doteqdot$ Department of Physics, University of Toronto, Toronto, Ontario, Canada M5S 1A?
}

Received 16 September 1986

\begin{abstract}
Using a vector coherent state theory, it is shown that the construction of Gel"fand bases for the unitary group is particularly simple. The very specific rules for construction of the states greatly facilitate the subsequent computation of matrix elements of the generators of the $u(n)$ Lie algebra.
\end{abstract}

\section{Introduction}

Our intention in the present series of papers is to exploit some recent developments in the theory of tensor representations of Lie groups to shed new light on the WignerRacah calculus for the unitary groups.

First, using a powerful vector coherent state theory (Rowe 1984, 1986, Rowe et al $1985 a$ ), it will be shown that the construction of Gel'fand bases for the unitary groups is particularly simple, this being largely due to the multiplicity-free decomposition of a given unitary irreducible representation of $u(n)$ into its $u(n-1)$ subgroup representations

$$
\mathrm{u}(n) \downarrow \mathrm{u}(n-1)
$$

(recall the Gel'fand pattern betweenness conditions). The very specific rules for construction of the states greatly facilitate the subsequent computation of matrix elements of the generators of the $\mathrm{u}(n)$ Lie algebra (Gel'fand and Tseitlin 1950) which is then seen to be a mere exercise in recoupling techniques.

Then, using to its full extent the principle of complementarity for boson space representations (Biedenharn et al 1967, Moshinsky 1963, Quesne 1986), the above coherent state theory, and the recent realisation (Le Blanc and Rowe 1986a, b) that a strict group theoretical meaning can be assigned to the Biedenharn-Louck $U(n)$ tensor operator patterns (see, e.g., Louck 1970) which permits the explicit construction of the elementary tensors, we rederive (Le Blanc and Hecht 1987, hereafter referred to as II) in a straightforward fashion the values of the elementary Wigner coefficients for the unitary groups (Biedenharn and Louck 1968). It will be shown there that the elementary Wigner calculus for $U(n)$ once more turns out to be an exercise in recoupling techniques. The explicit structure of the elementary Wigner coefficients will be given there and shown to consist of a product of a $U(n-1)$ Racah coefficient times some dimensional and (vector coherent state theory) normalisation factors. Each subcomponent is calculated on its own. The derivation is self-contained and appeals to a minimum number of group theoretical concepts and tools. 
Finally, in part III (Le Blanc 1987), we show that the framework adopted in II allows the computation in closed form of all $\mathrm{SU}(2)$ Wigner and Racah coefficients. We also derive there an interesting relationship between $U(3)$ symmetric tensors and U(2) Racah coefficients.

\section{Construction of Gel'fand bases}

\subsection{General remarks}

Let $g$ and $g^{c}$ denote an arbitrary Lie algebra and its complex extension, respectively. To apply vector coherent state theory to the evaluation of matrix elements of $g$, one must select a so-called 'core' subalgebra $\mathrm{k} \subset \mathrm{g}$ that contains the Cartan subalgebra and is such that $\mathrm{g}^{\mathrm{c}}$ can be decomposed:

$$
\mathrm{g}^{\mathrm{c}}=\mathrm{n}_{+}+\mathrm{k}^{\mathrm{c}}+\mathrm{n}_{-}
$$

where $\mathrm{n}_{ \pm}$are nilpotent subalgebras of raising and lowering operators, respectively.

Note that, like the familiar Cartan decomposition, such a decomposition is reductive in the sense that

$$
\begin{aligned}
& k \in \mathrm{k}, n_{+} \in \mathrm{n}_{+} \rightarrow\left[k, n_{+}\right] \in \mathrm{n}_{+} \\
& k_{1}, k_{2} \in \mathrm{k} \rightarrow\left[k_{1}, k_{2}\right] \in \mathrm{k} .
\end{aligned}
$$

It follows that the elements of $n_{ \pm}$can be regarded as components of tensor operators under the subalgebra $\mathrm{k}$. Consequently, the reduction (2.1) facilitates the decomposition of ladder representations of $g$ into irreducible representations of $k$. For example, it is easily shown that the subspace of states in an irreducible ladder representation of $g$ that satisfy

$$
n_{+}|\psi\rangle=0 \quad \forall n_{+} \in \mathrm{n}_{+}
$$

carries an irreducible representation of $\mathrm{k}$. We refer to this subspace as the highest weight $\mathrm{k}$ space. Similarly, the subspace of states that satisfy

$$
n_{-}|\psi\rangle=0 \quad \forall n_{-} \in \mathrm{n}_{-}
$$

spans an irreducible lowest weight $\mathrm{k}$ space. Application of the raising tensors to this lowest weight $\mathrm{k}$ space, or the lowering operators to the highest weight $\mathrm{k}$ space, then generates intermediate weight $k$ spaces. This generalises the familiar Cartan construction of raising and lowering from the lowest and highest weight states.

A major simplification arises when the operators in $n_{+}$(or $n_{-}$) are all commuting components of a single irreducible $\mathrm{k}$ tensor. For example, the first application of this theory (Rowe 1984) was to the symplectic Lie algebra $\operatorname{sp}(3, \mathfrak{H})$. In that application, $\mathrm{k}$ was put equal to the maximal compact subalgebra $u(3)$ and it was observed that the raising operators were the components of an irreducible $u(3)$ tensor of rank $\{200\}$. Thus, it became possible to classify the basis states for $\operatorname{sp}(3, \mathfrak{M})$ ladder representations

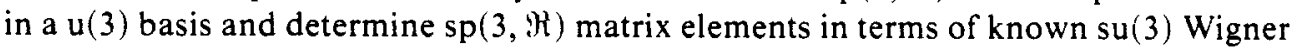
and Racah coefficients. It was also possible to determine the $\operatorname{sp}\left(n, \ell^{\ell}\right) \downarrow \mathrm{u}(n)$ branching rules for all ladder representations (Rowe et al 1985b).

In application to $\mathrm{u}(n)$, we naturally choose $\mathrm{k}=\mathrm{u}(n-1) \times \mathrm{u}(1)$ as core algebra. Then, as shown explicitly in the following subsection, the raising operators of $n_{+}$are components of a $u(n-1) \times u(1)$ tensor of rank $\{1 \dot{0}\} \times\{1\}$, where a dot over a digit is 
to signify its repetition, in this case $(n-2)$ times to complete the $\mathrm{u}(n-1)$ partition $\{10 \ldots 0\}$. Thus the quadratics of raising operators are $u(n-1) \times u(1)$ tensors of rank $\{2 \dot{0}\} \times\{2\}$ while, more generally, tensors of rank $w$ in the raising operators span a symmetrical irreducible representation $\{w \dot{0}\} \times\{w\}$ of $\mathrm{u}(n-1) \times \mathrm{u}(1)$.

Now, if $\{\lambda\}$ denotes an irreducible $u(n)$ ladder representation containing lowest and highest weight $\mathrm{u}(n-1)$ representations $\left\{\sigma_{\mid w}\right\}$ and $\left\{\sigma_{h w^{\prime}}\right\}$, respectively, then the familiar $\mathrm{u}(n) \downarrow \mathrm{u}(n-1)$ Gel'fand branching rules for $\{\lambda\}$ can be expressed:

$$
\{\lambda\} \downarrow \sum_{w=0,1, \ldots}\{w \dot{0}\} \times\left\{\sigma_{l w}\right\}
$$

with the understanding that representations not contained in $u(n)$ vanish identically. In the actual construction, this will be ensured by the vanishing of either the Racah coefficients $(2.30)$ or of the normalisation factor $K(2.23)$. Alternatively, since the lowering operators of $\mathrm{n}_{-}$are components of an irreducible $\mathrm{u}(n-1)$ tensor of rank $\{\dot{0}-1\}$, we have

$$
\{\lambda\} \downarrow \sum_{w=0,1, \ldots}\{\dot{0}-w\} \times\left\{\sigma_{h w}\right\} .
$$

There are two essential points. First, this decomposition exhibits explicitly the construction of a $\mathrm{u}(n-1)$ basis for a $\mathrm{u}(n)$ ladder representation by either stepping up from the lowest weight $\mathrm{u}(n-1)$ representation or stepping down from the highest weight representation with $\mathrm{u}(n-1)$ tensor raising or lowering operators, respectively. Second, this $\mathrm{u}(n-1)$ basis is multiplicity free. As a consequence of these properties, vector coherent state theory gives analytical expressions for the matrix elements of the algebra in terms of $\mathrm{u}(n-1)$ Wigner and Racah coefficients. Proceeding recursively, we thus obtain the matrix elements of $\mathrm{u}(n)$ in the familiar Gel'fand basis.

\subsection{Vector coherent state theory}

Given an abstract $\mathrm{u}(n)$ Lie algebra

$$
\left\{E_{\alpha \beta} ; 1 \leqslant \alpha, \beta \leqslant n\right\}
$$

with commutator algebra

$$
\left[E_{\alpha \beta}, E_{\mu \nu}\right]=\delta_{\beta \mu} E_{\alpha \nu}-\delta_{\alpha \nu} E_{\mu \beta}
$$

we decompose it into a $\mathrm{u}(n-1)$ subalgebra

$$
\left\{C_{i j}=E_{i j} ; 1 \leqslant i, j \leqslant n-1\right\}
$$

a weight operator

$$
W=E_{n n}
$$

with eigenvalue $m_{n n}^{0}+w$ (see equation (2.25)), a set of raising operators

$$
\left\{A_{i}=E_{\text {in }} ; 1 \leqslant i \leqslant n-1\right\}
$$

and a set of lowering operators

$$
\left\{B_{i}=E_{n} ; 1 \leqslant i \leqslant n-1\right\} \text {. }
$$


From $(2.2 b)$, we obtain the following set of commutation relations:

$$
\begin{aligned}
& {\left[C_{i l}, C_{k l}\right]=\delta_{j k} C_{i l}-\delta_{i l} C_{k l}} \\
& {\left[C_{i j}, A_{k}\right]=\delta_{j k} A_{i} \quad\left[C_{i j}, B_{k}\right]=-\delta_{i k} B_{l}} \\
& {\left[A_{i}, B_{j}\right]=C_{i j}-\delta_{i j} W} \\
& {\left[A_{i}, A_{j}\right]=\left[B_{i}, B_{j}\right]=\left[C_{i j}, W\right]=0} \\
& {\left[W, B_{i}\right]=B_{i} \quad\left[W, A_{i}\right]=-A_{i} .}
\end{aligned}
$$

Given a generic irreducible ladder representation $\left\{m^{0}\right\}_{n}$ of $\mathrm{u}(n)$, a $\mathrm{u}(n-1)$ highest weight space is defined by all the states belonging to the maximal $\mathrm{u}(n-1)$ subrepresentation $\left\{m^{0}\right\} \equiv\left\{m^{0}\right\}_{n-1}$; i.e. the $\mathrm{u}(n-1)$ subrepresentation with

$$
m_{i, n-1}^{0}=m_{i n}^{0} \quad 1 \leqslant i \leqslant n-1 .
$$

Let $\{|\eta\rangle\}$ denote an orthonormal but otherwise arbitrary basis for this highest weight $\mathrm{u}(n-1)$ representation. A vector coherent state representation $\Gamma(\mathscr{O})$ of an operator $\mathcal{O} \in \mathrm{u}(n)$ acts on the Hilbert space of vector-valued holomorphic functions defined by

$$
\langle z \mid \psi\rangle=\sum_{\eta}|\eta\rangle\left\langle\eta\left|\mathrm{e}^{z \cdot A}\right| \psi\right\rangle
$$

(Rowe et al 1985a) where $|\psi\rangle$ is any state of the unirrep $\left\{m^{0}\right\}_{n}$,

$$
z \cdot A=\sum_{i=1}^{n-1} z_{i} A_{i}
$$

and $z_{i}$ are $(n-1)$ complex (Bargmann) variables used as coordinates for the factor space

$$
\mathrm{U}(n) / \mathrm{U}(n-1) \text {. }
$$

Such a coherent state representation is given by

$$
\begin{aligned}
\Gamma(\mathcal{O})\langle z \mid \psi\rangle & =\sum_{\eta}|\eta\rangle\left\langle\eta\left|\mathrm{e}^{z \cdot A} \mathcal{O}\right| \psi\right\rangle \\
& =\sum_{\eta}|\eta\rangle\left\langle\eta\left|\left(\mathrm{e}^{z \cdot A} \mathcal{O} \mathrm{e}^{-z \cdot A}\right) \mathrm{e}^{z \cdot A}\right| \psi\right\rangle \\
& =\sum_{\eta}|\eta\rangle\left\langle\eta\left|\left(\mathcal{O}+\frac{1}{1 !}[z \cdot A, \mathcal{O}]+\frac{1}{2 !}[z \cdot A,[z \cdot A, \mathcal{O}]]+\ldots\right) \mathrm{e}^{z \cdot A}\right| \psi\right\rangle .
\end{aligned}
$$

Using (2.4) and (2.9), we find the following expansions (with the usual convention concerning the summation of repeated indices):

$$
\begin{aligned}
& \Gamma\left(A_{i}\right)=\partial / \partial z_{1} \equiv \partial_{i} \\
& \Gamma\left(C_{i j}\right)=\mathscr{E}_{i j}-z_{j} \partial_{i} \\
& \Gamma\left(B_{1}\right)=z_{k} \mathscr{E}_{k 1}-z_{1} \mathscr{E}_{n n}-z_{i} z_{k} \partial_{k} \\
& \Gamma(W)=\mathscr{E}_{n n}+z_{1} \partial_{1} .
\end{aligned}
$$

The set of operators

$$
\left\{\mathscr{E}_{i j}, 1 \leqslant i, j \leqslant n-1\right\}
$$

spans an intrinsic $\mathrm{u}(n-1)$ Lie algebra acting only on the highest weight space $\{|\eta\rangle\}$ while the set

$$
\left\{\mathscr{C}_{i j}=-z_{j} \partial_{i}, 1 \leqslant i, j \leqslant n-1\right\}
$$


spans a simple Weil (1964) (or oscillator) $\mathrm{u}(n-1)$ Lie algebra. Note that the set of operators $\left\{z_{l}, \partial_{,}, \delta_{l}, 1 \leqslant i, j \leqslant n-1\right\}$ close upon a Heisenberg-Weyl (boson) algebra $h w(n-1)$ with

$$
\left[\partial_{i}, z_{1}\right]=\delta_{11}
$$

Since $A_{i}$ carries a u $(n-1)$ irrep $\{1 \dot{0}\}, \partial_{i}$, through $(2.10 a, b)$, will also carry a $\{1 \dot{0}\}$ irrep of $\mathrm{u}(n-1)$. Thus $z_{i}$ carries a $\mathrm{u}(n-1)$ irrep $\{\dot{0}-1\}$ (see equation $(2.21 a)$ ).

The vector coherent state expansion $(2.10)$ is not unitary with respect to the usual boson Hermitian conjugation

$$
(z)^{\dagger}=\left(\frac{\partial}{\partial z}\right)
$$

and will be referred to as a Dyson expansion for the $\mathrm{u}(n)$ Lie algebra. In order to convert it into a unitary (Holstein-Primakoff) expansion, one must perform a $\mathrm{u}(n-1)$ invariant Hermitian transformation $K^{\dagger}=K$ such that the realisation $\gamma(\mathcal{O})$ defined by

$$
\gamma(\mathcal{C})=K^{-1} \Gamma(\mathcal{O}) K
$$

becomes unitary, i.e.

$$
\begin{aligned}
& {\left[\gamma\left(A_{i}\right)\right]^{\dagger}=\gamma\left(B_{i}\right)} \\
& {\left[\gamma\left(C_{i j}\right)\right]^{\dagger}=\gamma\left(C_{j i}\right) .}
\end{aligned}
$$

The use of $(2.10 a, c)$ and of the commutator identity (Rowe 1984)

$$
\Gamma\left(B_{i}\right)=\left[\Lambda, z_{i}\right]
$$

where

$$
\Lambda=\left(z_{l} \partial_{j}\right)\left(\mathscr{E}_{i j}-\delta_{l j} \mathscr{E}_{n n}\right)-\frac{1}{2}\left(z_{l} \partial_{j}\right)\left(z_{j} \partial_{l}\right)+\frac{1}{2}(n-1)\left(z_{k} \partial_{k}\right)
$$

is a $\mathrm{u}(n-1) \times \mathrm{u}(1)$ invariant operator, leads to

$$
\Gamma\left(B_{i}\right)=K^{2} z_{i} K^{-2}=\left[\Lambda, z_{i}\right]
$$

which can be used to solve for the operat is $K$. Since the various $u(n-1)$ representations $\{m\} \equiv\{m\}_{n-1}$ in the $\mathrm{u}(n)$ representations $\left\{m^{0}\right\}_{n}$ arise in the multiplicity-free fashion, the $K$ operator is diagonal in a $\mathrm{u}(n) \supset \mathrm{u}(n-1)$ basis with eigenvalues given by the recursion formula

$$
K^{2}\left(\left\{m^{\prime}\right\}\right) / K^{2}(\{m\})=\Omega\left(\left\{m^{\prime}\right\}\right)-\Omega(\{m\})
$$

where, for simplicity of notation, we suppress the $\left\{m^{0}\right\}_{n}$ dependence and the subscript $n-1$ of

$$
K^{2}\left(\begin{array}{c}
\left\{m^{0}\right\}_{n} \\
\{m\}_{n-1}
\end{array}\right)
$$

and

$$
\Omega\left(\begin{array}{c}
\left\{m^{0}\right\}_{n} \\
\{m\}_{n-1}
\end{array}\right) .
$$

These will be reintroduced in II since there we use tensors which, unlike the generators $A, B$ and $C$, connect different $\mathrm{u}(n)$ unirreps. 
In (2.19), $\Omega(\{m\})$ is the eigenvalue of the $u(n-1) \times u(1)$ invariant $\Lambda$ on the $u(n-1)$ coupled orthonormal basis defined by

$$
\langle z \mid\{m\} \eta\rangle \equiv\left[Z^{\left\{\dot{0}-n^{\prime}\right\}}(z) \times\left|\left\{m^{0}\right\}\right\rangle\right]_{\{m\} \eta}
$$

where the square bracket represents a $u(n-1)$ coupling which is defined more precisely in equation (2.26). The boson tensor $Z^{\{0-w \mid}(z)$ in $(2.20)$ is a symmetric polynomial in the Bargmann variables $z_{t}$ which carry, as seen earlier, a $\{\dot{0}-1\}$ unirrep of the $u(n-1)$ Lie algebra $(2.10 b)$. Its normalisation is fixed by its highest weight component

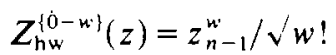

which readily implies that

$$
\left[Z^{\left\{\dot{0}-w_{1}\right\}}(z) \times Z^{\left\{\dot{0}-w_{2}\right\}}(z)\right]_{\{\dot{0}-w\}}=\delta_{w, w_{1}+w_{2}}\left(\frac{w !}{w_{1} ! w_{2} !}\right)^{1 / 2} Z^{\left\{\dot{0}-w^{\prime}\right\}}(z) .
$$

The partitions $\left\{m^{\prime}\right\}$ and $\{m\}$ in (2.19) can differ by at most minus one in, say, the $r$ th entry and we find

$$
\Omega\left(\left\{m-\Delta^{(1)}(r)\right\}\right)-\Omega(\{m\})=p_{r, n-1}-p_{n n}
$$

where

$$
p_{i j}=m_{i j}+j-i
$$

is the well known hook of the representation theory of the symmetric group (Robinson 1961) and

$$
\Delta^{(1)}(r)=(00 \ldots 010 \ldots 0)
$$

is a null $(n-1)$-vector except for its $r$ th entry.

Using (2.19) and (2.22), we then find

$$
K^{2}(\{m\})=\prod_{i=1}^{n-1} \frac{\left(p_{i n}-p_{n n}-1\right) !}{\left(p_{t, n-1}-p_{n n}\right) !}
$$

with

$$
K^{2}\left(\left\{m^{0}\right\}\right)=1
$$

if the highest weight state is properly normalised.

From the unitary preserving isomorphism $B_{i} \sim \gamma\left(B_{i}\right)=K z_{i} K^{-1}$, we finally obtain an orthonormal $\mathrm{u}(n) \supset \mathrm{u}(n-1)$ basis

$$
|\{m\} \eta\rangle \equiv\left|\begin{array}{c}
\left\{m^{0}\right\}_{n} \\
\{m\}_{n-1} \\
\eta
\end{array}\right|=\left[Z^{\langle 0-w\}}\left(K^{-1} B K\right) \times\left|\left\{m^{0}\right\}\right\rangle\right]_{\{m\} \eta} .
$$

The rank $w$ of the operator $Z$ in $(2.24)$ is given by

$$
w=\sum_{i=1}^{n-1} m_{i n}^{0}-\sum_{i=1}^{n-1} m_{l, n-1}
$$

and is equal to the eigenvalue of the weight operator $E_{n n}$ on states belonging to the $\mathrm{u}(n-1)$ irreducible space $\{m\}$ minus its eigenvalue on states belonging to the highest weight space $\left\{m^{0}\right\}$ :

$$
\begin{aligned}
w & =\left\langle E_{n n}\right\rangle-\left\langle E_{n n}\right\rangle_{h w} \\
& =\left\langle E_{n n}\right\rangle-m_{n n}^{0} .
\end{aligned}
$$


Note, however, that the phase of the square root $K$ of $K^{2}$ is arbitrary. We shall choose it such that the reduced matrix elements of the raising operators $A$ are all positive (see remark following (2.34)). We then obtain

$$
\begin{aligned}
&|\{m\} \eta\rangle=(-1)^{\phi\left(\left\{m^{0}\right\}\right)-\phi(\{\dot{0}-w\})-\phi(\{m\})} K^{-1}(\{m\})\left[Z^{\{0-w\}}(B) \times\left|\left\{m^{0}\right\}\right\rangle\right]_{\{m\} \eta} \\
&=(-1)^{\phi\left(\left\{m^{0}\right\}\right)-\phi(\{0-w\})-\phi(\{m\})} K^{-1}(\{m\}) \\
& \times \sum_{\eta_{1}, \eta_{2}}\left\langle\left\{m^{0}\right\} \eta_{1} ;\{\dot{0}-w\} \eta_{2} \mid\{m\} \eta\right\rangle Z_{\eta_{2}}^{\{\dot{0}-w\}}(B) \times\left|\left\{m^{0}\right\} \eta_{1}\right\rangle
\end{aligned}
$$

where $K(\{m\})$ is now defined to be the positive square root of $K^{2}(\{m\})$ and the $u(n-1)$ phase $\phi\left(\{m\}_{n-1}\right)$ is defined in the appendix. The quantity

$$
\left\langle\left\{m^{0}\right\} \eta_{1} ;\{\dot{0}-w\} \eta_{2} \mid\{m\} \eta\right\rangle
$$

in $(2.26 b)$ is an $\operatorname{SU}(n-1)$ Wigner coefficient.

Note the specific order of the coupling in $(2.26 b)$. This order has been chosen so that the usual form of the Wigner-Eckart theorem would apply directly to this and similar equations i.e. the tensor, here the polynomial $Z(B)$, acting on the initial state corresponds to the second entry in the Wigner coefficient. For ease of notation, we will often ignore in the following the basis labels $\eta$ on both sides of an equation like $(2.26 a)$. In such cases, it is understood that the respective labels do match.

\subsection{Reduced matrix elements of the generators}

In computing matrix elements of the generators of the $\mathrm{u}(n)$ Lie algebra, we assume that matrix elements of the $\mathrm{u}(n-1)$ subalgebra $(2.3 a)$ are known. This recursive approach then allows us to concentrate on the matrix elements for the raising and lowering operators $A$ and $B$ as defined by $(2.3 c)$ and $(2.3 d)$.

Since $A$ and $B$ are respectively $\{1 \dot{0}\}$ and $\{\dot{0}-1\}$ tensors under $\mathrm{u}(n-1)$, their matrix elements are products of a fundamental $S U(n-1)$ Wigner coefficient of the $\{1 \dot{0}\}$ or $\{\dot{0}-1\}$ kind, respectively, and a $u(n-1)$ reduced matrix element. These fundamental Wigner coefficients have been calculated by Baird and Biedenharn (1963). We will compute them in II in a more structured and straightforward way which will make use of the present results. Here we concentrate on the computation of the reduced matrix elements themselves.

The $\mathrm{u}(n-1)$ reduced matrix element for $B$ is defined by

$$
\left\langle\left\{m^{\prime}\right\}\|B\|\{m\}\right\rangle=\sum_{\eta_{1}, \eta_{2}}\left\langle\{m\} \eta_{1} ;\{\dot{0}-1\} \eta_{2} \mid\left\{m^{\prime}\right\} \eta\right\rangle\left\langle\left\{m^{\prime}\right\} \eta\left|B_{\eta_{2}}\right|\{m\} \eta_{1}\right\rangle .
$$

Introducing the expansion $(2.26 b)$ in $(2.27)$, we can perform a $\mathrm{U}(n-1)$ recoupling in order to reunite the two terms in the operator $B$. Application of equation $(2.21 b)$ yields

$$
\left[B \times Z^{\{\dot{0}-w\}}(B)\right]_{\{\dot{0}-(n+1)\}}=\sqrt{w+1} Z^{\{\dot{0}-(w+1)\}}(B) .
$$

We thus find that

$$
\begin{aligned}
& \left\langle\left\{m-\Delta^{(1)}(r)\right\}\|B\|\{m\}\right\rangle \\
& =(-1)^{\phi(\{m\})-\phi\left(\left\{m-د^{(1)}(r)\right\}\right)-\phi(\{\dot{0}-\{x+1)\})+\phi(\{\dot{0}-x\})} \sqrt{w+1} \\
& \times U\left(\left\{m^{0}\right\}\{\dot{0}-w\}\left\{m-\Delta^{(1)}(r)\right\}\{\dot{0}-1\} ;\{m\}\{\dot{0}-(w+1)\}\right) \\
& \times K\left(\left\{m-\Delta^{(1)}(r)\right\}\right) / K(\{m\})
\end{aligned}
$$


where $U\left(\left\{m^{0}\right\}\{\dot{0}-w\}\left\{m-\Delta^{(1)}(r)\right\}\{\dot{0}-1\} ;\{m\}\{\dot{0}-(w+1)\}\right)$ is a $U(n-1)$ multiplicityfree Racah coefficient which can be obtained through permutation group techniques (see appendix 2 of II, and also Kramer (1967), Hecht (1975)). We show in the appendix of II that

$$
\begin{aligned}
U\left(\left\{m^{0}\right\}\{\dot{0}-w\right. & \left.\left\{m-\Delta^{(1)}(r)\right\}\{\dot{0}-1\} ;\{m\}\{\dot{0}-(w+1)\}\right) \\
= & U\left(\left\{m-\Delta^{(1)}(r)\right\}\{1 \dot{0}\}\left\{m^{0}\right\}\{w \dot{0}\} ;\{m\}\{(w+1) \dot{0}\}\right) \\
= & \left(\frac{1}{w+1} \prod_{j=1}^{n-1} \frac{p_{r, n-1}-p_{j n}}{p_{r, n-1}-p_{j, n-1}-1}\right)^{1 / 2} .
\end{aligned}
$$

(Conjugation properties of the Racah coefficients are also discussed in the appendix.)

Thus, using the expressions $(2.19 a)$ for the $K$ ratio, equation (2.30) for the Racah coefficient and expression (A2) to simplify the phase in (2.29), we find that the reduced matrix element of $B$ is given by

$$
\begin{aligned}
& \left\langle\left\{m-\Delta^{(1)}(r)\right\}\|B\|\{m\}\right\rangle \\
& \quad=(-1)^{\phi(\{m\})-\phi(\{\dot{0}-1\})-\phi\left(\left\{m-\Delta^{(1)}(r)\right\}\right)}\left(\frac{\prod_{k=1}^{n}\left(p_{r, n-1}-p_{k n}\right)}{\prod_{k=1}^{n-1}\left(p_{r, n-1}-p_{k, n-1}-1\right)}\right)^{1 / 2} .
\end{aligned}
$$

Reduced matrix elements of $A$ are obtained through $\mathrm{U}(n-1)$ conjugation and we find (see the appendix for conjugation properties of unitary tensors)

$$
\begin{aligned}
\left\langle\left\{m+\Delta^{(1)}(r)\right\}\right. & \|A\|\{m\}\rangle \\
= & (-1)^{\phi(\{m\})+\phi(\{10\})-\phi\left(\left\{m+J^{(1)}(r)\right\}\right)}\left(\frac{\operatorname{dim}\left(\{m\}_{n-1}\right)}{\operatorname{dim}\left(\left\{m+\Delta^{(1)}(r)\right\}_{n-1}\right)}\right)^{1 / 2} \\
& \times\left\langle\{m\}\|B\|\left\{m+\Delta^{(1)}(r)\right\}\right\rangle \\
= & \left(\frac{\prod_{k=1}^{n}\left(p_{r, n-1}-p_{k n}+1\right)}{\prod_{k=1}^{n-1}\left(p_{r, n-1}-p_{k, n-1}+1\right)}\right)^{1 / 2}
\end{aligned}
$$

where $\operatorname{dim}\left(\{m\}_{n}\right)$ is the dimension of the $U(n)$ unirrep $\{m\}_{n}$ and is given by the Weyl (1931) formula

$$
\operatorname{dim}\left(\{m\}_{n}\right)=\frac{\prod_{r<<=1}^{n}\left(p_{r n}-p_{\llcorner n}\right)}{1 ! 2 ! \ldots(n-1) !}
$$

We see from equation ( $2.32 b$ ) that all reduced matrix elements of $A$ are positive. We thus respect the generally accepted convention of imparting positive reduced matrix elements to the generators corresponding to the positive roots of the Lie algebra (generators of positive rank). In particular, it can be verified that

$$
\langle\{1 \dot{0}\}\|\boldsymbol{A}\|\{0\}\rangle=+1
$$

which implies that all components of the fundamental (n-vector) unirrep $\{1 \dot{0}\}$ of $U(n)$ are positive.

\section{Appendix. Phase conventions}

The purpose of this appendix is to state unambiguously the phase conventions to be used in this series of papers. A prime criterion in our choice of phases is that all $\mathrm{U}(n)$ 
conventions must reduce to the well known Condon-Shortley conventions for $U(2)$ when $n=2$.

We first seek to generalise the quantity

$$
\phi\left(\left\{f_{1} f_{2}\right\}\right)=\frac{1}{2}\left(f_{1}-f_{2}\right) \equiv j .
$$

We therefore introduce the following functional:

$$
\begin{aligned}
\phi\left(\{f\}_{n}\right) & =\sum_{i=j}^{n} \frac{1}{2}\left(f_{i}-f_{j}\right) \\
& =\frac{1}{2} \sum_{i=1}^{n}(n+1-2 i) f_{i}
\end{aligned}
$$

defined on a generic $U(n)$ partition. It has the following properties.

(1) It reduces to the well known $\mathrm{U}(2)$

$$
j=\frac{1}{2}\left(f_{1}-f_{2}\right)
$$

and U(3) (Hecht 1965)

$$
\lambda+\mu=\left(f_{1}-f_{3}\right)
$$

equivalent quantities.

(2) It is invariant under Hermitian conjugation

$$
f_{i} \rightarrow \tilde{f}_{i}=-f_{n-i+1} .
$$

(3) The quantity $4 \phi\left(\{f\}_{n}\right)$ is always even and $\phi$ thus admits the existence of spinor representations.

(4) A conjugation expression like

$$
\phi\left(\left\{f^{(1)}\right\}_{n}\right)+\phi\left(\left\{f^{(2)}\right\}_{n}\right)-\phi\left(\left\{f^{(3)}\right\}_{n}\right)
$$

is always an integer and is actually independent of $n$, i.e. this expression is only row (and not $\mathrm{U}(n)$ ) dependent when considered in terms of Young tableaux.

We next seek to generalise the Hermitian conjugation factor $\omega(j, m)=j-m$ of SU(2). Since we do not need an explicit expression for $\omega$, which depends on the basis subgroup decomposition and the state construction, but only its fundamental properties, we therefore only enumerate the latter.

(5) The conjugation factor $\omega\left(\{f\}_{n}, m\right)$, where $m$ here is a $\mathrm{U}(n-1) \supset \mathrm{U}(n-2) \supset$ $\ldots \supset \mathrm{U}(1)$ Gel'fand pattern, must be an integer and therefore $2 \omega\left(\{f\}_{n}, m\right)$ is even.

(6) Also, we must have

$$
\begin{aligned}
& \omega\left(\{f\}_{n}, m\right)+\omega\left(\{\tilde{f}\}_{n}, \tilde{m}\right)=2 \phi\left(\{f\}_{n}\right) \\
& \begin{array}{c}
\omega\left(\left\{f^{(1)}\right\}_{n}, m^{(1)}\right)+\omega\left(\left\{f^{(2)}\right\}_{n}, m^{(2)}\right)-\omega\left(\left\{f^{(3)}\right\}_{n}, m^{(3)}\right) \\
=\phi\left(\left\{f^{(1)}\right\}_{n}\right)+\phi\left(\left\{f^{(2)}\right\}_{n}\right)-\phi\left(\left\{f^{(3)}\right\}_{n}\right)
\end{array}
\end{aligned}
$$

where $\tilde{m}_{i j}=-m_{j-1+1, j}$.

We now require the following conjugation properties for the full (not reduced) $\mathrm{U}(n)$ multiplicity-free Wigner coefficients:

$$
\begin{aligned}
& \left\langle f^{(1)} m^{(1)} ; f^{(2)} m^{(2)} \mid f^{(3)} m^{(3)}\right\rangle=(-1)^{\phi\left(\left\{f^{(1)}\right)_{n}\right)+\phi\left(\left\{f^{(2)}\right\}_{11}\right)-\phi\left(\left\{f^{(3)}\right\}_{n}\right)} \\
& \times\left\langle f^{(2)} m^{(2)} ; f^{(1)} m^{(1)} \mid f^{(3)} m^{(3)}\right\rangle \\
& \left\langle f^{(1)} m^{(1)} ; f^{(2)} m^{(2)} \mid f^{(3)} m^{(3)}\right\rangle=(-1)^{\phi\left(\left\{f^{(1)}\right\}_{n}\right)+\phi\left(\left\{f^{(2)}\right\}_{n}\right)-\phi\left(\left\{f^{(1)}\right\}_{n}\right)} \\
& \times\left\langle\tilde{f}^{(1)} \tilde{m}^{(1)} ; \tilde{f}^{(2)} \tilde{m}^{(2)} \mid \tilde{f}^{(3)} \tilde{m}^{(3)}\right\rangle
\end{aligned}
$$




$$
\begin{aligned}
\left\langle f^{(1)} m^{(1)} ; f^{(2)}\right. & m^{(2)}\left|f^{(3)} m^{(3)}\right\rangle \\
= & (-1)^{\phi\left(\left\{f^{(1)}\right\}_{n}\right)+\phi\left(\left\{f^{(2)}\right\}_{n}\right)-\phi\left(\left\{f^{(3)}\right\}_{n}\right)-\omega\left(\left\{f^{(2)}\right\}_{n}, m^{(2)}\right)} \\
& \times\left(\frac{\operatorname{dim}\left\{f^{(3)}\right\}_{n}}{\operatorname{dim}\left\{f^{(1)}\right\}_{n}}\right)^{1 / 2}\left\langle f^{(3)} m^{(3)} ; \tilde{f}^{(2)} \tilde{m}^{(2)} \mid f^{(1)} m^{(1)}\right\rangle .
\end{aligned}
$$

With the help of the preceding conjugation properties for the Wigner coefficients, it is then easy to prove various conjugation properties of the $u(n)$ multiplicity-free Racah coefficients like equation (2.30) using the defining equation

$$
\begin{aligned}
U\left(\left\{f^{(1)}\right\}\left\{f^{(2)}\right\}\right. & \left.\{f\}\left\{f^{(3)}\right\} ;\left\{f^{(12)}\right\}\left\{f^{(23)}\right\}\right) \\
= & \frac{1}{\operatorname{dim}\{f\}_{n}} \sum_{\text {all } m^{\prime}}\left\langle\left\{f^{(1)}\right\} m^{(1)} ;\left\{f^{(2)}\right\} m^{(2)}\right|\left\{f^{(12)}\right\} m^{(12)} \\
& \times\left\langle\left\{f^{(12)}\right\} m^{(12)} ;\left\{f^{(3)}\right\} m^{(3)} \mid\{f\} m\right\rangle \\
& \times\left\langle\left\{f^{(2)}\right\} m^{(2)} ;\left\{f^{(3)}\right\} m^{(3)} \mid\left\{f^{(23)}\right\} m^{(23)}\right\rangle \\
& \times\left\langle\left\{f^{(1)}\right\} m^{(1)} ;\left\{f^{(23)}\right\} m^{(23)} \mid\{f\} m\right\rangle .
\end{aligned}
$$

Finally, reduced matrix elements of a $\mathrm{U}(n)$ tensor $\mathcal{O}^{\left\{f^{(2)}\right\}_{n}}$ are related to the reduced matrix elements of its Hermitian conjugate $\mathcal{O}^{\left\{f^{(2)}\right\}_{n}}$ through

$$
\begin{aligned}
\left\langle\left\{f^{(3)}\right\}_{n}\left\|\mathcal{O}^{\left\{f^{(2)}\right\}_{n}}\right\|\left\{f^{(1)}\right\}_{n}\right\rangle & \\
= & (-1)^{\phi\left(\left\{f^{(1)}\right\}_{n}\right)+\phi\left(\left\{f^{(2)}\right\}_{n}\right)-\phi\left(\left\{f^{(3)}\right\}_{n}\right)}\left(\frac{\operatorname{dim}\left\{f^{(1)}\right\}_{n}}{\operatorname{dim}\left\{f^{(3)}\right\}_{n}}\right)^{1 / 2} \\
& \times\left\langle\left\{f^{(1)}\right\}_{n}\left\|\mathcal{O}^{\left\{f^{(2)}\right\}_{n}}\right\|\left\{f^{(3)}\right\}_{n}\right\rangle .
\end{aligned}
$$

\section{References}

Baird G E and Biedenharn L C 1963 J. Math. Phys, 41443

Biedenharn L C, Giovanninni A and Louck J D 1967 J. Math. Phys. 8691

Biedenharn L C and Louck J D 1968 Commun. Math. Phys. 889

Gel'fand I M and Tseitlin M L 1950 Dokl. Akad. Nauk. 71 825, 1017

Hecht K T 1965 Nucl. Phys. 621

1975 Commun. Math. Phys. 41135

Kramer P 1967 Z. Phys. 205181

Le Blanc R 1987 J. Phys. A: Math. Gen. submitted

Le Blane R and Hecht K T 1987 J. Phys. A: Math. Gen. submitted

Le Blanc R and Rowe D J 1986a J. Phys. A: Math. Gen. 191093

— 1986b J. Phys. A: Math. Gen. 192913

Louck J D 1970 Am. J. Phys. 383

Moshinsky M 1963 J. Math. Phys. 41128

Quesne C 1986 J. Phy's. A: Math. Gen. 192689

Robinson G de B 1961 Representation Theory of the Symmetric Group (Toronto: University of Toronto Press) Rowe D J 1984 J. Math. Phys. 252662

_- 1986 Phase Space Approach to Nuclear Dynamics ed M di Toro, W Norenberg, M Rosina and S Stringani (Singapore: World Scientific) p 546

Rowe D J, Rosensteel G and Gilmore R 1985a J. Math. Phys. 262787

Rowe D J, Wybourne B G and Butler P H 1985b J. Phys. A: Math. Gen. 18939

Weil A 1964 Acta Math. 111143

Weyl H 1931 The Theory of Groups and Quantum Mechanics (New York: Dover) 